\title{
Efficacy and Safety of Afatinib for EGFR-mutant Non-small Cell Lung Cancer, Compared with Gefitinib or Erlotinib
}

\author{
Youjin Kim, MD \\ Se-Hoon Lee, MD, PhD \\ Jin Seok Ahn, MD, PhD \\ Myung-Ju Ahn, MD, PhD \\ Keunchil Park, MD, PhD \\ Jong-Mu Sun, MD, PhD
}

Division of Hematology-Oncology, Department of Internal Medicine, Samsung Medical Center, Sungkyunkwan University School of Medicine, Seoul, Korea
Correspondence: Jong-Mu Sun, MD, PhD Division of Hematology-Oncology, Department of Medicine, Samsung Medical Center, Sungkyunkwan University School of Medicine, 81 Irwon-ro, Gangnam-gu,

Seoul 06351, Korea

Tel: 82-2-3410-1795

Fax: 82-2-3410-1754

E-mail: jongmu.sun@skku.edu

Received February 14, 2018

Accepted June 10, 2018

Published Online June 13, 2018

\begin{abstract}
Purpose
We tried to evaluate whether there are any specific features in treatment outcomes of firstline afatinib in patients with epidermal growth factor receptor (EGFR)-mutant non-small cell lung cancer (NSCLC), compared with gefitinib or erlotinib.
\end{abstract}

\section{Materials and Methods}

We analyzed patients treated with first-line afatinib, gefitinib, or erlotinib for advanced EGFRmutant NSCLC at Samsung Medical Center between 2014 and 2016.

\section{Results}

In total, 467 patients received first-line afatinib $(n=165)$, gefitinib $(n=230)$, or erlotinib $(n=72)$. Afatinib was used more often in patients with tumors harboring deletion in exon 19 (Del19), whereas the gefitinib group had more elderly, females, and never smokers. The median progression-free survival (PFS) time for afatinib, gefitinib, and erlotinib was 19.1 months, 13.7 months, and 14.0 months, respectively $(p=0.001)$. The superior PFS of afatinib was more remarkable in subgroups of Del19 or uncommon EGFR mutations. Overall toxicity profiles of the three drugs were comparable, though more grade 3 or 4 toxicities were detected in afatinib (7.3\%) compared with gefitinib (2.6\%) or erlotinib (1.8\%). The common grade 3 or 4 toxicities of afatinib included diarrhea (3.0\%), paronychia (2.4\%), and skin rash (1.8\%). Dose modification was more frequently required in patients treated with afatinib (112/165, 68\%), compared with gefitinib (5/230, 2\%) and erlotinib (4/72, 6\%). Interestingly, however, dose reduction in the afatinib group did not impair its efficacy in terms of PFS (dose reduction vs. no reduction group, 23.5 months vs. 12.4 months).

\section{Conclusion}

First-line afatinib showed satisfactory efficacy data and manageable toxicity profiles.

\section{Introduction}

Non-small cell lung cancer (NSCLC) is one of the leading causes of deaths from cancer worldwide [1]. When used as first-line therapy, first-generation epidermal growth factor receptor (EGFR) tyrosine kinase inhibitors (TKIs) such as gefitinib and erlotinib have improved clinical outcomes in patients with advanced NSCLC harboring EGFR mutations $[2,3]$.

\section{Key words}

Afatinib, First-line therapy, Epidermal growth factor receptor, Non-small cell lung carcinoma
Afatinib, a second-generation EGFR TKI, is an oral, irreversible ErbB family blocker that selectively and potently blocks signaling from all relevant ErbB family receptors (ErbB1, ErbB2, and ErbB4) [4]. In the Phase III LUX-Lung 3 and LUX-Lung 6 trials, first-line afatinib significantly improved progression-free survival (PFS) and objective response rates versus platinum-doublet chemotherapy in patients with EGFR-mutant NSCLC [5,6]. These promising results led to the approval of afatinib in many countries from 2013 onwards. 
Several large randomized trials have been conducted to compare the clinical efficacies and toxicities of these EGFR TKIs. The WJOG 5108L study was a randomized phase III study comparing gefitinib with erlotinib. It did not demonstrate statistical non inferiority of gefitinib in terms of PFS compared with erlotinib [7]. The LUX-Lung 7 trial compared afatinib with gefitinib as a first-line treatment in patients with advanced NSCLC harboring common EGFR mutations (Del19 and the L858R point mutation) [8]. Although the median PFS times of afatinib and gefitinib were similar (11.0 months vs. 10.9 months), afatinib showed a statistically superior PFS outcome compared to gefitinib (hazard ratio [HR], 0.73; 95\% confidence interval [CI], 0.57 to 0.95 ) [8].

Currently, afatinib, gefitinib, and erlotinib are recommended as first-line therapies for EGFR-mutant NSCLC. However, no rules or guidelines are available to choose one of these drugs for a given patient. Thus, the specific drug is typically chosen according to the physician's preference or experience. In South Korea, these three TKIs are approved, and one of three TKIs can be chosen by physicians as the first-line therapy for their patients with EGFR-mutant NSCLC.

A few years have elapsed since first-line afatinib therapy for EGFR-mutant NSCLC was first reimbursed in Korea (October 2014). Our purpose of this study is to investigate how afatinib is used in real-world practice, and whether it leads to different clinical outcomes compared with gefitinib or erlotinib.

\section{Materials and Methods}

\section{Patients}

This retrospective population-based study included patients with NSCLC who received first-line afatinib, gefitinib, or erlotinib at a single institute (Samsung Medical Center) between October 2014 and December 2016. Eligible patients in the clinical database of the hospital were identified and their medical information was extracted from electronic medical records. Patient demographics such as age, sex, smoking history, performance status, and EGFR mutation type were reviewed. Demographic information was obtained for the time at which first-line EGFR TKI treatment was initiated. EGFR mutations were identified using a PNA clamp kit and real-time polymerase chain reaction. Mutations other than Del19 or the L858R point mutation were classified as uncommon EGFR mutations, which also included the de novo T790M mutation. In the efficacy analysis according to EGFR mutation type, the T790M mutation was exclu- ded since tumors harboring T790M are known to be resistant to afatinib, gefitinib, and erlotinib.

\section{Evaluation of safety and efficacy}

All patients were diagnosed with NSCLC by histologic analysis. Tumor stage was evaluated by chest computed tomography (CT), positron-emission tomography-CT (PET$\mathrm{CT}$ ), and brain magnetic resonance imaging (MRI). If the results of staging work-up procedures performed in hospitals other than Samsung Medical Center were of poor quality, we repeated the procedures at our hospital before the patients started treatment.

When starting first-line EGFR TKIs, all patients were informed about the potential adverse events of the TKIs and instructed on how to manage such events if they appear. All patients were recommended to visit the clinic within 2 weeks after starting TKIs so that they could be screened for any adverse events. If any significant adverse event had occurred by the first visit after TKI treatment, dose reduction or a more vigilant follow-up schedule was planned. Otherwise, patients visited every 1 or 2 months for the first 6 months, and every 2 or 3 months thereafter. Tumor responses were checked every 2 or 3 months by chest CT, with or without abdomen/pelvis CT, PET-CT, or brain MRI.

Objective response rate was calculated by checking the best response according to the Response Evaluation Criteria in Solid Tumor ver. 1.1 guidelines. PFS was defined as the time from starting first-line TKI to disease progression or death; overall survival was defined as the time from starting first-line TKI to death. Toxicity was graded according to the National Cancer Institute Common Toxicity Criteria, ver. 4.0.

\section{Statistical considerations}

To compare baseline characteristics among the three groups or treatments, one-way ANOVA or the chi-square test was used. Overall survival was defined as the time from registration to death from any cause or censored at the time of last contact. Progression-free survival (PFS) was defined as the time from registration to documented progression or death without progression. Patients without documented progression or death were censored at the time of the last disease assessment. The survival data were analyzed using the Kaplan-Meier method. Median PFS was calculated using a Cox proportional hazards model. PFS is presented as median values and two-sided 95\% CIs. PFS after gefitinib, erlotinib, or afatinib treatment was estimated with a Cox proportional hazards model. For multivariable analysis, a multiple Cox regression model was fitted by adjusting significant prognostic variables $(p<0.05)$ in the univariable analysis for PFS. All $p$-values were two-sided and a $\mathrm{p}<0.05$ was considered 
Table 1. Baseline characteristics

\begin{tabular}{|c|c|c|c|c|}
\hline Patient characteristic & Afatinib & Gefitinib & Erlotinib & p-value \\
\hline No. of patients & 165 & 230 & 72 & \\
\hline \multicolumn{5}{|l|}{ Age (yr) } \\
\hline Median (range) & $57(30-79)$ & $64(29-87)$ & $59(36-77)$ & $<0.001$ \\
\hline$<60$ & $93(56.4)$ & $79(34.3)$ & $38(52.8)$ & $<0.001$ \\
\hline$\geq 60$ & $72(43.6)$ & $151(65.7)$ & $34(47.2)$ & \\
\hline \multicolumn{5}{|l|}{ Sex } \\
\hline Male & $85(51.5)$ & $60(26.1)$ & $39(54.2)$ & $<0.001$ \\
\hline Female & $80(48.5)$ & $170(73.9)$ & $33(45.8)$ & \\
\hline \multicolumn{5}{|l|}{ ECOG PS } \\
\hline 0 & $42(25.5)$ & $56(24.3)$ & $24(33.3)$ & 0.658 \\
\hline 1 & $114(69.0)$ & $160(69.6)$ & $44(61.1)$ & \\
\hline 2 & $9(5.5)$ & $14(6.1)$ & $4(5.6)$ & \\
\hline \multicolumn{5}{|l|}{ Smoking status } \\
\hline Never smoker & $99(60.0)$ & $180(78.3)$ & $41(56.9)$ & $<0.001$ \\
\hline Current or ex-smoker & $66(40.0)$ & $50(21.7)$ & $31(43.1)$ & \\
\hline \multicolumn{5}{|l|}{ EGFR mutation type } \\
\hline Exon 19 deletion & $114(69.1)$ & $122(53.0)$ & $40(55.6)$ & 0.002 \\
\hline Exon 21 L858R & $37(22.4)$ & $96(41.8)$ & $27(37.5)$ & \\
\hline Uncommon EGFR & $14(8.5)$ & $12(5.2)$ & $5(6.9)$ & \\
\hline
\end{tabular}

Values are presented as median (range) or number (\%). Uncommon EGFR: the tumor contains a mutation other than del19 or L858R. ECOG, Eastern Cooperative Oncology Group; PS, performance status; EGFR, epidermal growth factor receptor.

statistically significant. Data were analyzed using SPSS ver. 17 (SPSS Inc., Chicago, IL). The data cutoff for the analysis was May 28, 2017.

\section{Ethical statement}

This study was reviewed and approved by the institutional review board (IRB No. 2018-02-111) at Samsung Medical Center. The informed consent was waived because this study was done based on the medical record review. The trial was conducted in accordance with the Declaration of Helsinki.

\section{Results}

\section{Patient characteristics and preference for afatinib, gefi- tinib, or erlotinib}

The patient characteristics are summarized in Table 1. A total of 467 patients with recurrent or metastatic stage IV NSCLC were included in the analysis, each of whom had Del19, L858R point mutation, or an uncommon EGFR mutation. Patients were treated with afatinib $(n=165)$, gefitinib $(\mathrm{n}=230)$, or erlotinib $(\mathrm{n}=72)$ as the first-line therapy. Patients in the gefitinib group were significantly elderly (median age, 64 years) than those in the afatinib and erlotinib groups (median age, 57 and 59 years, respectively) $(\mathrm{p}<0.001)$. Female patients comprised less than $50 \%$ of the afatinib and erlotinib groups, while $74 \%$ of the gefitinib group was female $(\mathrm{p}<0.001)$. Performance status was not significantly different among the three groups. The proportion of never smokers was higher in the gefitinib group $(78 \%)$ than in the afatinib $(60 \%)$ and erlotinib groups $(57 \%)(\mathrm{p}<0.001)$. Approximately $70 \%$ of patients in the afatinib group had Del19; this percentage was significantly higher than those in the gefitinib (53\%) and erlotinib $(56 \%)$ groups ( $\mathrm{p}=0.002)$. A total of 31 patients with uncommon EGFR mutation types were included, broken down by treatment group as follows: afatinib $(\mathrm{n}=14)$, gefitinib ( $n=12)$, and erlotinib $(n=5)$.

\section{Survival outcomes according to EGFR TKI therapy}

The median follow-up duration for PFS was 17.7 months (95\% CI, 16.5 to 18.9). The median PFS times for afatinib, gefitinib, and erlotinib were 19.1 months (95\% CI, 12.3 to 25.9), 13.7 months (95\% CI, 12.3 to 15.1$)$, and 14.0 months $(95 \% \mathrm{CI}$, 11.3 to 16.8 ), respectively ( $\mathrm{p}=0.001$ ) (Fig. $1 \mathrm{~A}$ ). In the univariate analysis for PFS, other clinical characteristics such as common EGFR mutation type ( $<<0.001)$, good performance status (Eastern Cooperative Oncology Group 0 or 1$)(p<0.001)$, 

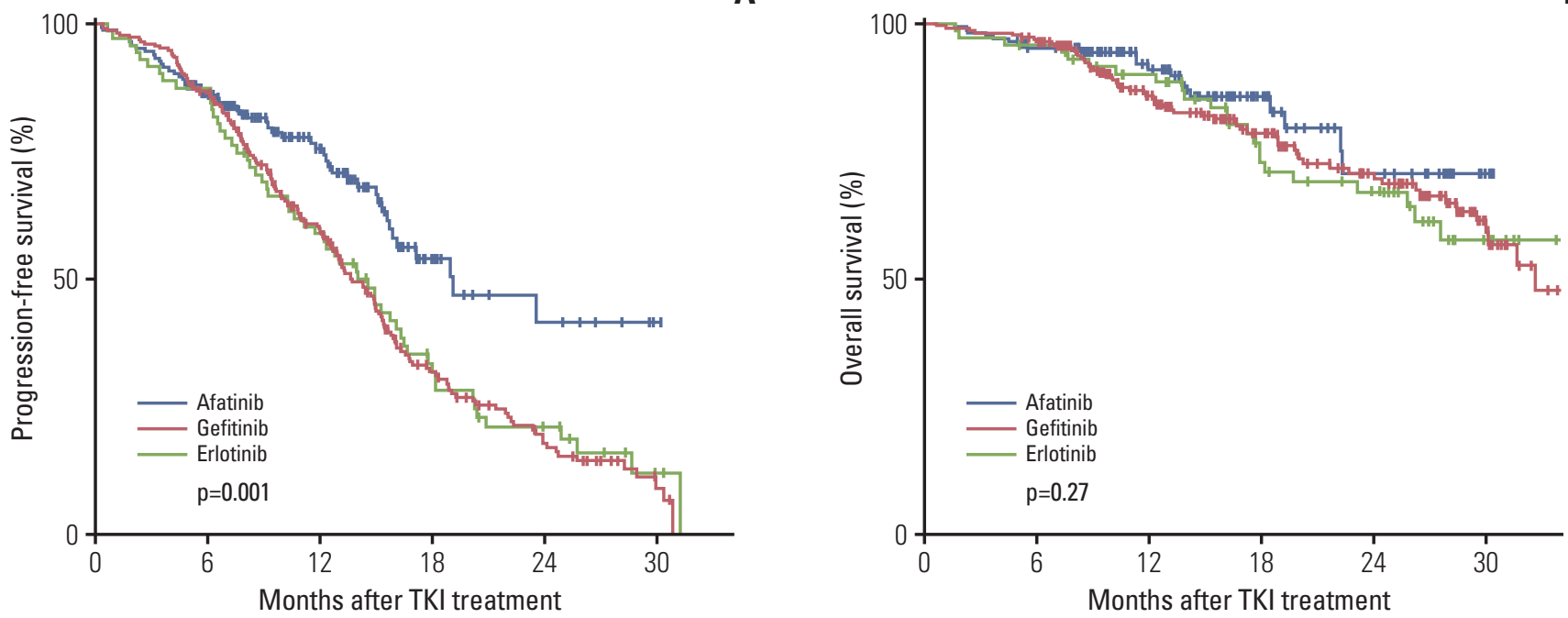

Fig. 1. Progression-free survival (A) and overall survival (B) with afatinib, gefitinib, and erlotinib. TKI, tyrosine kinase inhibitor.

and never smoker status $(\mathrm{p}=0.014)$ were associated with longer PFS, while younger age $(<60$ years $)(\mathrm{p}=0.174)$ and female sex $(\mathrm{p}=0.523)$ were not good prognostic factors in terms of predicting PFS. With respect to the other clinical factors considered, afatinib therapy was significantly associated with longer PFS in the multivariate analysis (HR, 0.46; $95 \%$ CI, 0.34 to $0.63 ; \mathrm{p}<0.001)$.

Regarding overall survival, the median follow-up duration was 17.5 months (95\% CI, 16.3 to 18.8). There was no statistically significant difference between the EGFR TKI therapy groups ( $\mathrm{p}=0.21$ ) (Fig. 1B).

\section{Progression-free survivals of EGFR TKIs according to EGFR mutation types}

We next analyzed PFS according to EGFR mutation types. In the subgroup of patients with Del19, the median PFS times for afatinib, gefitinib, and erlotinib were 19.1 months, 15.0 months, and 16.3 months, respectively $(\mathrm{p}=0.01)$. In contrast, there was no significant difference in the L858R subgroup $(\mathrm{p}=0.46)$ (Fig. 2A and B). For the subgroup of uncommon EGFR mutations, the afatinib group showed much longer PFS (median PFS, not reached) compared with the gefitinib (5.0 months) and erlotinib (6.1 months) groups, though it was not statistically significant due to a small sample size ( $\mathrm{p}=0.06)$ (Fig. 2C).

\section{Objective response in the uncommon EGFR mutation subgroup}

Table 2 shows the uncommon EGFR mutation types and the objective response of patients with each mutation type to each EGFR TKI. A total of 31 patients had uncommon mutations (exon 21 L861 Q, exon 18 G719X, exon 20 insertion, exon 18 G719X+exon 20 S768I, Del19+L747_P753>Q, and exon 21 L858R+H870R) and seven patients (1.4\%) had both active and resistant EGFR mutations (T790M). Seven patients had the de novo T790M mutation (afatinib, 4; gefitinib, 3); these patients had no response. Among the patients harboring uncommon mutations other than T790M, objective response was seen in eight out of the 10 patients in the afatinib group, while four out of nine in the gefitinib group showed a response and one out of five in the erlotinib group showed a response.

\section{Toxicity profile}

The toxicity profiles were compatible with the expectation (Table 3). The most common adverse events were skin rash, stomatitis, paronychia, and diarrhea. The incidence of grade 1-2 adverse events were comparable among three treatment groups. However, grade 3-4 adverse events were more frequently found in the afatinib group $(7.3 \%)$, compared with gefitinib $(2.6 \%)$ and erlotinib groups $(1.8 \%)$. The common grade 3-4 adverse events of afatinib were diarrhea, paronychia, and skin rash. A total of 10 patients of afatinib permanently discontinued afatinib therapy due to grade 2 parony- 
A


C

Fig. 2. Progression-free survival of afatinib, gefitinib, and erlotinib according to epidermal growth factor receptor (EGFR) mutation types. (A) Exon 19 deletion. (B) Exon 21 L858R. (C) Uncommon EGFR mutations. TKI, tyrosine kinase inhibitor.

chia or skin rash $(n=3)$, grade 2 or 3 diarrhea $(n=4)$, grade 2 or 3 stomatitis $(n=2)$, or grade 3 pneumonitis $(n=1)$. One patient with gefitinib hold permanently due to grade 3 interstitial lung disease, and there was no permanent hold in the erlotinib group. No treatment-related deaths occurred for all the three drugs.

\section{Dose modification}

All patients initiated afatinib, gefitinib, and erlotinib treatment with $40 \mathrm{mg}$, $250 \mathrm{mg}$, and $150 \mathrm{mg}$ once daily, respec- 
Table 2. Objective response rates according to uncommon EGFR mutation

\begin{tabular}{|c|c|c|c|c|c|c|}
\hline \multirow[b]{2}{*}{ EGFR TKI } & \multicolumn{2}{|c|}{ Afatinib } & \multicolumn{2}{|c|}{ Gefitinib } & \multicolumn{2}{|c|}{ Erlotinib } \\
\hline & Total & $\begin{array}{l}\text { Objective } \\
\text { response }\end{array}$ & Total & $\begin{array}{l}\text { Objective } \\
\text { response }\end{array}$ & Total & $\begin{array}{l}\text { Objective } \\
\text { response }\end{array}$ \\
\hline Uncommon EGFR mutation & 14 & 8 & 12 & 4 & 5 & 1 \\
\hline Uncommon EGFR mutation other than T790M & 10 & 8 & 9 & 4 & 5 & 1 \\
\hline \multicolumn{7}{|l|}{ Uncommon $E G F R$ mutation } \\
\hline Exon 21 L858R+exon 20 T790M & 3 & 0 & 3 & 0 & 0 & 0 \\
\hline Exon 19 deletion+exon 20 T790M & 1 & 0 & 0 & 0 & 0 & 0 \\
\hline Exon 21 L861 Q & 3 & 3 & 4 & 2 & 0 & 0 \\
\hline Exon 18 G719X & 3 & 2 & 4 & 2 & 3 & 1 \\
\hline Exon 20 insertion & 1 & 0 & 0 & 0 & 2 & 0 \\
\hline Exon 18 G719X+exon 20 S768I & 1 & 1 & 1 & 0 & 0 & 0 \\
\hline Exon 19 Deletion+L747_P753>Q & 1 & 1 & 0 & 0 & 0 & 0 \\
\hline Exon 21 L858R+H870R & 1 & 1 & 0 & 0 & 0 & 0 \\
\hline
\end{tabular}

EGFR, epidermal growth factor receptor; TKI, tyrosine kinase inhibitor.

Table 3. Toxicity profile of afatinib, gefitinib, and erlotinib

\begin{tabular}{|c|c|c|c|c|c|c|}
\hline \multirow{2}{*}{ Toxicity } & \multicolumn{3}{|c|}{ Grade 1-2 } & \multicolumn{3}{|c|}{ Grade 3-4 } \\
\hline & Afatinib & Gefitinib & Erlotinib & Afatinib & Gefitinib & Erlotinib \\
\hline Total & $116(70.3)$ & $164(71.3)$ & $53(73.6)$ & $12(7.3)$ & $6(2.6)$ & $3(1.8)$ \\
\hline Rash and acne & $76(46.1)$ & $131(57.0)$ & $47(65.3)$ & $3(1.8)$ & $1(1.4)$ & $3(1.8)$ \\
\hline Stomatitis & $50(30.3)$ & $31(13.5)$ & $8(11.1)$ & 0 & 0 & 0 \\
\hline Paronychia & $44(26.7)$ & $21(9.1)$ & $13(18.1)$ & $4(2.4)$ & 0 & 0 \\
\hline Diarrhea & $32(19.4)$ & $33(14.3)$ & $13(18.2)$ & $6(3.0)$ & 0 & 0 \\
\hline Dry skin & $18(10.9)$ & $45(19.6)$ & $12(16.7)$ & 0 & $1(1.4)$ & 0 \\
\hline Pruritus & $17(10.3)$ & $79(34.3)$ & $21(29.2)$ & 0 & 0 & 0 \\
\hline Anorexia & $7(4.2)$ & $15(6.5)$ & $7(9.7)$ & $1(0.6)$ & $1(1.4)$ & 0 \\
\hline Nausea/Vomiting & 0 & $4(1.7)$ & $1(1.4)$ & 0 & 0 & 0 \\
\hline Fatigue & $1(0.6)$ & $3(1.3)$ & $1(1.5)$ & $1(0.6)$ & 0 & 0 \\
\hline Alopecia & 0 & $15(6.5)$ & $2(2.8)$ & 0 & $1(1.4)$ & 0 \\
\hline Interstitial lung disease & 0 & 0 & 0 & $1(0.6)$ & 0 & 0 \\
\hline Increased AST/ALT & 0 & $2(0.9)$ & 0 & 0 & $2(2.8)$ & 0 \\
\hline
\end{tabular}

Values are presented as number (\%). ALT, alanine aminotransferase; AST, aspartate aminotransferase.

increasing the administration interval into every other day, due to skin toxicity and hepatotoxicity. Four patients with erlotinib reduced dose to $100 \mathrm{mg}$ per day due to skin toxicity. A total of 10 patients in the afatinib group permanently discontinued afatinib therapy due to grade 2 paronychia or skin rash $(n=3)$, grade 2 or 3 diarrhea $(n=4)$, grade 2 or 3 stomatitis $(n=2)$, or grade 3 pneumonitis $(n=1)$. One patient with gefitinib permanently stopped medication due to skin toxicity and there was no permanent stop case for erlotinib. No treatment-related deaths occurred for all three TKIs.

We analyzed whether dose reduction of afatinib affected its efficacy. Interestingly, in the survival analysis according to final afatinib dose, reduction did not impair PFS: the median PFS times for the unreduced group $(40 \mathrm{mg})$ and reduced group ( $30 \mathrm{mg}$ or $20 \mathrm{mg}$ ) were 12.4 months and 23.5 months, respectively (S1 Fig.). When the disease burdens were compared by initial brain metastasis status between two groups, there was no significant difference. There were 19 patients (35\%) with brain metastasis in the unreduced group $(\mathrm{n}=55)$, while 48 patients ( $43 \%)$ initially presented with metastatic brain tumor in the reduced group $(n=112)(p=0.53)$. 


\section{Discussion}

To our knowledge, this is the first study to comprehensively evaluate the pattern of clinical use of three EGFR TKIs (afatinib, gefitinib, and erlotinib). We found that gefitinib was more commonly used than erlotinib or afatinib in Korea, especially for elderly, which may be due to concerns about treatment related adverse events. A previous retrospective report showed that gefitinib was less associated with significant skin toxicity than the other two TKIs in patients with NSCLC [9]. Compatible with this report, our study also showed that gefitinib was prescribed more often to women, indicating that physicians consider potential cosmetic problems when treating women. Afatinib was more commonly used for patients with tumors harboring Del19. We reason that this observation could be due to a study reporting that the afatinib arm had longer overall survival than the chemotherapy arm in the Del19 subgroup, while this result was not observed in the L858R subgroup [10].

Our results show that afatinib was significantly associated with longer PFS compared with gefitinib and erlotinib, even after adjustment for other potentially confounding prognostic factors. In addition, the significantly longer PFS of afatinib observed even in the Del19 subgroup could preclude the possibility that longer PFS of afatinib for overall population was caused by that more patients with better prognostic factor, Del19, were included in the afatinib group. We confirm the previous data that showed the superior PFS outcome of second-generation EGFR TKIs such as afatinib and dacomitinib to first-generation EGFR TKI such as gefitinib $[8,11]$.

The median PFS times of each TKI in our study (13-19 months) were longer than those (11 months) reported from prospective trials as first-line EGFR TKIs $[5,6,8]$. Although we do not have a definitive explanation for this result, maintaining TKI treatment for a long-term period with more efficient management of adverse events may in part contribute to the longer PFS. We note that the rate of total grade 3 or 4 adverse events for afatinib, gefitinib, and erlotinib were approximately $7 \%, 3 \%$, and $2 \%$, respectively, in our study, which is lower than those reported in prospective studies [5-8].

Although gefitinib and erlotinib have both demonstrated efficacy for treating tumors with common EGFR mutations [12-14], their efficacy against tumors with uncommon EGFR mutations is still under investigation. The response rates and median PFS of first-generation EGFR TKIs for uncommon EGFR mutations were reported to be less than $50 \%$ and 5 months, respectively $[15,16]$. Recently, good efficacy of afatinib for treating tumors with uncommon mutations was reported $[17,18]$. Our study confirms the previous efficacy data of afatinib for NSCLC harboring uncommon EGFR mutations other than T790M. Compared with gefitinib and erlotinib, afatinib treatment yielded a longer PFS for patients with uncommon EGFR mutations, although this difference did not reach statistical significance, possibly due to the small sample size $(p=0.06)$. Based on our results, we recommend afatinib for first-line therapy in patients harboring uncommon EGFR mutations other than the de novo T790M point mutation.

The frequency of adverse events was lower than in previous prospective studies and fewer types of adverse events were observed $[5,6,8]$. This finding could be related to limitations commonly associated with retrospective studies, which are based on medical records. Despite these limitations, we observed a lower rate of significant toxicity (grade 3 or 4 ). This finding can be explained by the fact that our practice usually includes dose reduction with or without temporary interruption, in addition to appropriate use of oral antibiotics, anti-diarrheal agents, or steroid ointments for the management of adverse events.

In our study, more patients underwent dose reduction due to adverse events compared to previous prospective trials $[5,6,8]$. However, this dose reduction did not impair efficacy outcomes in terms of PFS. Therefore, based on our results, we recommend that physicians reduce the afatinib dose when patients experience unacceptable or prolonged adverse events. However, the relationship between dose reduction and PFS should be interpreted with caution, because there are more opportunities for dose reduction when patients take afatinib for longer periods. However, this bias was unlikely to have significantly affected the results because most dose reduction occurred soon after starting afatinib treatment, with a median time of 4.9 weeks (95\% CI, 4.0 to 5.8). In addition, reduced afatinib doses were shown to not impair efficacy in another retrospective study [19]. It showed that there was no difference in time to treatment failure between the group that started afatinib $40 \mathrm{mg}$ and the group that started $30 \mathrm{mg}$ as the first-line therapy for NSCLC [19].

In summary, we evaluated the frequencies of first-line EGFR TKI use in patients with EGFR-mutant NSCLC and found that patient characteristics affected the choice of EGFR TKI. Afatinib showed superior PFS data compared with gefitinib or erlotinib. Afatinib showed more grade 3 or 4 adverse events than gefitinib or erlotinib, though the incidence was much lower than previous data.

\section{Electronic Supplementary Material}

Supplementary materials are available at Cancer Research and Treatment website (https:// www.e-crt.org). 


\section{Conflicts of Interest}

Conflict of interest relevant to this article was not reported.

\section{Acknowledgments}

We thank all participating patients and their families, as well as the research nurses and study coordinators.

\section{References}

1. Torre LA, Bray F, Siegel RL, Ferlay J, Lortet-Tieulent J, Jemal A. Global cancer statistics, 2012. CA Cancer J Clin. 2015;65:87108.

2. Mok TS, Wu YL, Thongprasert S, Yang CH, Chu DT, Saijo N, et al. Gefitinib or carboplatin-paclitaxel in pulmonary adenocarcinoma. N Engl J Med. 2009;361:947-57.

3. Zhou C, Wu YL, Chen G, Feng J, Liu XQ, Wang C, et al. Erlotinib versus chemotherapy as first-line treatment for patients with advanced EGFR mutation-positive non-smallcell lung cancer (OPTIMAL, CTONG-0802): a multicentre, open-label, randomised, phase 3 study. Lancet Oncol. 2011;12: 735-42.

4. Li D, Ambrogio L, Shimamura T, Kubo S, Takahashi M, Chirieac LR, et al. BIBW2992, an irreversible EGFR/HER2 inhibitor highly effective in preclinical lung cancer models. Oncogene. 2008;27:4702-11.

5. Sequist LV, Yang JC, Yamamoto N, O'Byrne K, Hirsh V, Mok $\mathrm{T}$, et al. Phase III study of afatinib or cisplatin plus pemetrexed in patients with metastatic lung adenocarcinoma with EGFR mutations. J Clin Oncol. 2013;31:3327-34.

6. Wu YL, Zhou C, Hu CP, Feng J, Lu S, Huang Y, et al. Afatinib versus cisplatin plus gemcitabine for first-line treatment of Asian patients with advanced non-small-cell lung cancer harbouring EGFR mutations (LUX-Lung 6): an open-label, randomised phase 3 trial. Lancet Oncol. 2014;15:213-22.

7. Urata Y, Katakami N, Morita S, Kaji R, Yoshioka H, Seto T, et al. Randomized phase III study comparing gefitinib with erlotinib in patients with previously treated advanced lung adenocarcinoma: WJOG 5108L. J Clin Oncol. 2016;34:3248-57.

8. Park K, Tan EH, O'Byrne K, Zhang L, Boyer M, Mok T, et al. Afatinib versus gefitinib as first-line treatment of patients with EGFR mutation-positive non-small-cell lung cancer (LUXLung 7): a phase $2 \mathrm{~B}$, open-label, randomised controlled trial. Lancet Oncol. 2016;17:577-89.

9. Chen KL, Lin CC, Cho YT, Yang CW, Sheen YS, Tsai HE, et al. Comparison of skin toxic effects associated with gefitinib, erlotinib, or afatinib treatment for non-small cell lung cancer. JAMA Dermatol. 2016;152:340-2.

10. Yang JC, Wu YL, Schuler M, Sebastian M, Popat S, Yamamoto $\mathrm{N}$, et al. Afatinib versus cisplatin-based chemotherapy for EGFR mutation-positive lung adenocarcinoma (LUX-Lung 3 and LUX-Lung 6): analysis of overall survival data from two randomised, phase 3 trials. Lancet Oncol. 2015;16:141-51.
11. Mok T, Cheng Y, Zhou X, Lee KH, Nakagawa K, Niho S, et al. Dacomitinib versus gefitinib for the first-line treatment of advanced EGFR mutation positive non-small cell lung cancer (ARCHER 1050): a randomized, open-label phase III trial. J Clin Oncol. 2017;35(Suppl):LBA9007.

12. Jackman DM, Yeap BY, Sequist LV, Lindeman N, Holmes AJ, Joshi VA, et al. Exon 19 deletion mutations of epidermal growth factor receptor are associated with prolonged survival in non-small cell lung cancer patients treated with gefitinib or erlotinib. Clin Cancer Res. 2006;12:3908-14.

13. Lynch TJ, Bell DW, Sordella R, Gurubhagavatula S, Okimoto RA, Brannigan BW, et al. Activating mutations in the epidermal growth factor receptor underlying responsiveness of nonsmall-cell lung cancer to gefitinib. N Engl J Med. 2004;350: 2129-39.

14. Paez JG, Janne PA, Lee JC, Tracy S, Greulich H, Gabriel S, et al. EGFR mutations in lung cancer: correlation with clinical response to gefitinib therapy. Science. 2004;304:1497-500.

15. Wu JY, Shih JY. Effectiveness of tyrosine kinase inhibitors on uncommon E709X epidermal growth factor receptor mutations in non-small-cell lung cancer. Onco Targets Ther. 2016;9: 6137-45.

16. Baek JH, Sun JM, Min YJ, Cho EK, Cho BC, Kim JH, et al. Efficacy of EGFR tyrosine kinase inhibitors in patients with EGFRmutated non-small cell lung cancer except both exon 19 deletion and exon 21 L858R: a retrospective analysis in Korea. Lung Cancer. 2015;87:148-54.

17. Shen YC, Tseng GC, Tu CY, Chen WC, Liao WC, Chen WC, et al. Comparing the effects of afatinib with gefitinib or Erlotinib in patients with advanced-stage lung adenocarcinoma harboring non-classical epidermal growth factor receptor mutations. Lung Cancer. 2017;110:56-62.

18. Yang JC, Sequist LV, Geater SL, Tsai CM, Mok T, Schuler MH, et al. Activity of afatinib in uncommon epidermal growth factor receptor (EGFR) mutations: findings from three trials of afatinib in Egfr mutation-positive lung cancer. J Thorac Oncol. 2013;8(Suppl 2):S141.

19. Liu CY, Wang CL, Li SH, Hsu PC, Chen CH, Lin TY, et al. The efficacy of $40 \mathrm{mg}$ versus dose de-escalation to less than $40 \mathrm{mg}$ of afatinib (Giotrif) as the first-line therapy for patients with primary lung adenocarcinoma harboring favorable epidermal growth factor mutations. Oncotarget. 2017;8:97602-12. 